\title{
Concentration-effect relationships with carbamazepine and its epoxide on psychomotor and cognitive function in epileptic patients
}

\author{
R A GillhaM,* N Williams, * K WiEDMANN,* E BUTLER, $\dagger$ J G LARKIN, \\ M J BRODIE†
}

From the Department of Clinical Psychology, ${ }^{*}$ Institute of Neurological Sciences, Southern General Hospital, and Clinical Pharmacology Unit, $\dagger$ University Department of Medicine, Western Infirmary, Glasgow, Scotland

SUMMARY A battery of psychometric tests was administered to 85 patients with epilepsy, of whom 26 were untreated, 40 received carbamazepine monotherapy and 19 took carbamazepine with another anticonvulsant. Carbamazepine alone had little effect on performance, but carbamazepine polypharmacy produced significant impairment. Increasing concentrations of carbamazepine (four tests) and its active metabolite, carbamazepine 10,11 epoxide (seven tests), correlated with decreasing performance in the monotherapy patients.

Since the epidemiological studies of the 1950s, an association has been established between seizure disorders and neuropsychological deficit. ${ }^{12}$ The complex relationship between fits, cognitive impairment, psychosocial difficulties and underlying cerebral pathology has been the subject of several recent investigations. $^{3-5}$ The tangle of causality between these four factors has not been fully unravelled. Since the first controlled study of Reynolds and Travers, ${ }^{6}$ there has been a growing body of evidence that a fifth factor, the presence of antiepileptic drugs in the brain, contributes independently to disruption of intellectual functioning. Thompson and Trimble ${ }^{7}$ and Ludgate and his colleagues ${ }^{8}$ have shown that patients receiving multiple anticonvulsants function less well on cognitive testing than those treated with monotherapy and that reducing the number of circulating drugs can lead to improvement in performance without producing a deterioration in seizure control.

Patients taking carbamazepine appear to show less evidence of cerebral impairment than those treated with older agents such as phenytoin or phenobarbitone. $^{9-11}$ However, Macphee and co-workers, in a recent series of studies using a battery of simple psychomotor tests, have demonstrated that subtle derangement can be produced in naive subjects

Address for reprint requests: Dr Martin J Brodie, Clinical Pharmacology Unit, University Department of Medicine, Western Infirmary, Glasgow G11 6NT, Scotland, UK.

Received 22 January 1988

Accepted 15 March 1988 following a single dose of carbamazepine, ${ }^{12}$ in patients with epilepsy given a single supplementary dose ${ }^{13}$ and in patients on chronic carbamazepine monotherapy. ${ }^{14}$ The purpose of the present investigation was to examine more closely the relationship between carbamazepine, its active metabolite carbamazepine 10,11 epoxide (CBZ-E) and psychomotor and cognitive functioning in patients with epilepsy.

\section{Materials and methods}

\section{Patients}

Eighty-five patients attending the epilepsy clinic at the Western Infirmary were studied (table 1). Forty were taking carbamazepine alone and 19 received carbamazepine with one other anticonvulsant (8 sodium valproate, 6 phenytoin, 3 primidone, 2 phenobarbitone). Dosages had not been altered over the previous 3 months and all patients were compliant with their anticonvulsant medication as judged by consistent concentrations at out-patient attendances. No other drugs were being prescribed and no patient was known to abuse alcohol. Twenty-six untreated patients were included as controls. They were studied following an isolated seizure, during investigation of a likely seizure disorder or at least 6 months after a trial of drug withdrawal. The protocol had the approval of the Western Ethics Committee and all patients gave informed consent.

\section{Analyses}

Blood samples were taken from the treated patients at the time of testing and stored at $-20^{\circ} \mathrm{C}$ for batch analysis. Carbamazepine and CBZ-E concentrations were obtained using high performance liquid chromatography by a modification of the method outlined by Meijer. ${ }^{15}$ Lower 
Table 1 Clinical features in all patient groups

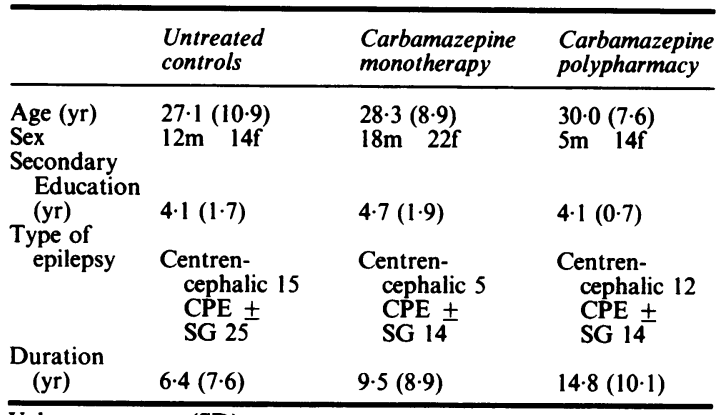

Values are mean (SD).

$\mathrm{CPE} \pm \mathrm{SG}=$ Complex partial epilepsy \pm secondary generalisation.

limit of detection for the CBZ-E assay was $0.2 \mathrm{mg} / \mathrm{l}$ which had a coefficient of variation of $<5 \%$.

\section{Test Battery}

The test battery was administered immediately after blood sampling in all patients. The average time for completion was 45 minutes. The following tests were performed:

(1) Performance IQ: Calculated from score on Ravens Standard Progressive Matrices.

(2) Decision Time: Time (ms) to respond to light coming on by removing finger from base button in choice reaction time task. A mean of 30 tests was recorded.

(3) Choice Reaction Time: Time (ms) to move finger from base button to extinguish light. A mean of 30 tests was recorded.

(4) Finger Tapping: Number of taps (per minute) of dominant index finger on calculator button in constant addition mode.

(5) Threshold Detection: An array of small rectangles is displayed on a VDU. After a very brief period of time an extra rectangle is added to the array. The subject is required to indicate which is the extra rectangle. The "threshold" is the minimum time gap in Frame Units between the presentation of the array and the additional stimulus which the subject requires to perceive that an extra rectangle has been added.

(6) Movement Detection: An array of small rectangles is displayed on a VDU. One moves out of position and back once. The subject is required to indicate which rectangle moved. Score is number of correct responses out of 32 .

(7) Forward Digit Span: Maximum number of digits subject can recall immediately following oral presentation. The subject is allowed two trials at each level and the task is discontinued when he fails both.

(8) Backward Digit Span: Maximum number of digits the subject can recall in reverse order immediately following oral presentation. The subject is allowed two trials at each level and the task is discontinued when he fails both.

(9) Paired Association Learning: Number of trials to reach criterion of three correct in learning unrelated word pairs.

(10) Visual Span: An array of boxes is displayed on a VDU. Some are unshaded and some shaded. The first array is replaced by a second after $100 \mathrm{~ms}$. In the second display one box which was previously shaded is now unshaded. The subject is required to indicate which box has changed. In the first example there are only two boxes. The task makes increasing demands on the subject's visual memory as more boxes are added. The visual span is the highest number of boxes in the display before the subject is unable to identify the change.

(11) Sedation Score: Subjects rating of level of alertness using $10 \mathrm{~cm}$ line where 0 is "nearly asleep" and 10 "wide awake".

(12) Side effect Score: The subject is presented with a standardised list of 15 side-effects associated with carbamazepine therapy (for example dizziness, diplopia, nausea, headache). Each one is rated on a four point scale as follows: $0=$ not present, $1=$ mild, $2=$ moderate, $3=$ severe. The individual ratings are summated to produce a side effect score.

Tests 2 and 3 were carried out using the choice reaction time facility of the Leeds Psychomotor Tester. Tests 5, 6 and 10 were developed in the Psychology Department at Stirling University and have been standardised using normal controls and alcoholic patients. They were administered using an Apple IIe microcomputer. Tests 7 and 8 are the Digit Span sub-test of Wechsler Memory Scale. Test 9 was the Inglis Paired Associate Learning test.

\section{Results}

There were no significant differences in age, in years of secondary education or in duration or type of epilepsy between the three groups (table 1, ANOVA). Patients taking more than one anticonvulsant drug had higher circulating levels of CBZ-E ( $t$ test, $p<0.01$ ) but mean carbamazepine concentrations in these two groups were almost identical (table 2).

Monthly seizure frequency and scores for each test are shown in table 3. ANOVA revealed significant differences between the groups on three tests: forward digit span $(p<0.05)$, sedation $(p<0.05)$ and side effect scores $(p<0.01)$. Multivariate analysis gave an $F$ value (Pillais) of 2.01 (df 2,26; $p<0.02$ ) showing the discriminative value of the test battery taken as a whole. When seizure frequency was taken out of this analysis as a covariate, the $F$ value remained significant at the $2 \%$ level ( $F$ value $1 \cdot 87$, df 2,26 ; $p<0.02$ ).

There was no difference in performance between patients on carbamazepine monotherapy and untreated patients, except with the movement detection task $(p<0.05)$. Patients treated with

Table 2 Concentrations (mean, $S D$ ) of carbamazepine and carbamazepine 10,11 epoxide (CBZ-E) in monotherapy and polypharmacy patients

\begin{tabular}{lll}
\hline & $\begin{array}{l}\text { Carbamazepine } \\
(\mathrm{mg} / \mathrm{l})\end{array}$ & $\begin{array}{l}\text { CBZ-E } \\
(\mathrm{mg} / \mathrm{l})\end{array}$ \\
\hline $\begin{array}{l}\text { Monotherapy } \\
(\mathrm{n}=40)\end{array}$ & $10.4(4.5)$ & $1.3(0.9)^{*}$ \\
$\begin{array}{l}\text { Polypharmacy } \\
(\mathrm{n}=19)\end{array}$ & $11.1(3.9)$ & $2.5(1.5)$ \\
\hline
\end{tabular}

Statistics obtained by $t$ test.

${ }^{*} \mathrm{p}<0.005$. 
Table 3 Mean (SD) seizure frequency and test scores in each group

\begin{tabular}{|c|c|c|c|}
\hline Test & $\begin{array}{l}\text { Epileptic } \\
\text { controls }\end{array}$ & $\begin{array}{l}\text { Carbamazepine } \\
\text { monotherapy }\end{array}$ & $\begin{array}{l}\text { Carbamazepine } \\
\text { polypharmacy }\end{array}$ \\
\hline $\begin{array}{l}\text { Seizures* } \\
\text { (per month) } \\
\text { Performance } \\
\text { IQ } \\
\text { Decision Time* } \\
\text { (ms) } \\
\text { Choice Reaction }\end{array}$ & $\begin{array}{l}1 \cdot 7 \\
(4 \cdot 3) \\
90 \cdot 6 \\
(17 \cdot 7) \\
0 \cdot 44 \\
(0 \cdot 11)\end{array}$ & $\begin{array}{l}9 \cdot 2 \dagger \\
(20 \cdot 0) \\
96 \cdot 5 \S \\
(17 \cdot 2) \\
0 \cdot 50 \\
(0 \cdot 23)\end{array}$ & $\begin{array}{l}10 \cdot 6 \ddagger \\
(8 \cdot 5) \\
85 \cdot 3 \\
(13 \cdot 2) \\
0 \cdot 61 \\
(0 \cdot 59)\end{array}$ \\
\hline $\begin{array}{l}\text { Time* } \\
\text { (ms) } \\
\text { Finger Tapping } \\
\text { (per min) } \\
\text { Threshold }\end{array}$ & $\begin{array}{l}0.72 \\
(0.21) \\
315 \cdot 8 \\
(91.4)\end{array}$ & $\begin{array}{l}0 \cdot 77 \\
(0.27) \\
314 \cdot 4 \\
(67 \cdot 9)\end{array}$ & $\begin{array}{l}0.92 \ddagger \\
(0.57) \\
294.0 \\
(73.0)\end{array}$ \\
\hline $\begin{array}{l}\text { Detection* } \\
\text { (Frame units) } \\
\text { Movement } \\
\text { Detection } \\
\text { Forward } \\
\text { Digit Span } \\
\text { Backward } \\
\text { Digit Span } \\
\text { Paired }\end{array}$ & $\begin{array}{l}4 \cdot 1 \\
(4 \cdot 5) \\
17 \cdot 3 \\
(9 \cdot 2) \\
6 \cdot 4 \\
(1 \cdot 3) \\
4 \cdot 9 \\
(1 \cdot 3)\end{array}$ & $\begin{array}{l}5 \cdot 2 \\
(5 \cdot 0) \\
11 \cdot 8 t \\
(10 \cdot 2) \\
6 \cdot 6 \$ \\
(1 \cdot 2) \\
4 \cdot 8 \S \\
(1 \cdot 6)\end{array}$ & $\begin{array}{l}6 \cdot 6 \\
(5 \cdot 2) \\
14 \cdot 6 \\
(9 \cdot 1) \\
5 \cdot 7 \\
(1 \cdot 3) \\
3 \cdot 9 \pm \\
(1 \cdot 1)\end{array}$ \\
\hline $\begin{array}{l}\text { Association } \\
\text { Learning* } \\
\text { Visual Span } \\
\text { Sedation Score } \\
\text { (cm) } \\
\text { Side Effect } \\
\text { Score* }\end{array}$ & $\begin{array}{l}4 \cdot 2 \\
(3 \cdot 1) \\
12 \cdot 0 \\
(4 \cdot 1) \\
7 \cdot 3 \\
(2 \cdot 0) \\
2 \cdot 8 \\
(3 \cdot 4)\end{array}$ & $\begin{array}{l}4 \cdot 8 \\
(4 \cdot 4) \\
12 \cdot 2 \\
(4 \cdot 5) \\
7 \cdot 1 \S \\
(2 \cdot 9) \\
3 \cdot 8 \S \\
(4 \cdot 6)\end{array}$ & $\begin{array}{l}6 \cdot 7 \ddagger \\
(4 \cdot 6) \\
11 \cdot 6 \\
(4 \cdot 6) \\
5 \cdot 3 \pm \\
(3 \cdot 1) \\
7 \cdot 1 \pm \\
(5 \cdot 5)\end{array}$ \\
\hline
\end{tabular}

* Denotes test where low score is better than high score.

t $t$ test shows control group better than Carbamazepine monotherapy group $\mathrm{p}<0.05$.

$\ddagger t$ test shows control group better than Carbamazepine polypharmacy group $\mathrm{p}<0.05$.

$\S t$ test shows CBZ monotherapy group better than Carbamazepine polypharmacy group $\mathrm{p}<0.05$.

carbamazepine and one other anticonvulsant did less well in choice reaction time, back digit span, paired association learning (all $p<0.05$ ) than the epileptic control group and in performance IQ and forward and back digit span (all $\mathrm{p}<0.05$ ) than the monotherapy patients. The polypharmacy group also reported greater sedation and side effects than the other patients (all $\mathrm{p}<0.05$ ).

Spearman correlations between carbamazepine and CBZ-E concentrations and test scores are outlined in table 4 . There were significant negative correlations for four tests with carbamazepine and for seven tests with CBZ-E. The relationships between carbamazepine and CBZ-E and choice reaction time and threshold detection are illustrated in figs 1 and 2 respectively. The poorer correlation between CBZ-E as a percentage of carbamazepine (two tests only) suggests that carbamazepine and CBZ-E exert independent effect on psychomotor function. Circulating levels of CBZ-E $(r=0.35, p=0.14)$ but not carbamazepine correlated positively with seizure frequency.

To compare directly performances of patients with high and low concentrations, the sample was divided into two groups; those with carbamazepine levels $<10.0 \mathrm{mg} / \mathrm{l}(\mathrm{n}=18)$ and those $>10.0 \mathrm{mg} / \mathrm{l}(\mathrm{n}=20)$. The high concentration patients scored significantly worse (Mann-Whitney U test) on threshold detection (p < 0.001), finger tapping ( $<<0.05)$, decision time $(p<0.05)$ and choice reaction time $(p<0.02)$. There was no significant difference between these two groups in terms of seizure frequency or duration of the disorder.

The sample was divided into two further groups; those with a CBZ-E concentration $<1.15 \mathrm{mg} / \mathrm{l}$ $(\mathrm{n}=19)$ and those $>1.15 \mathrm{mg} / \mathrm{l}(\mathrm{n}=19)$. The high concentration patients scored significantly worse on threshold detection $(\mathrm{p}<0.01)$, decision time $(p<0.05)$, and choice reaction time $(p<0.02)$. Once again, there was no significant difference in seizure frequency or duration of epilepsy.

\section{Discussion}

The three groups of patients in this study were not absolutely matched for seizure frequency or duration of epilepsy. Not surprisingly also, both the carbamazepine groups reported a higher seizure frequency and longer duration of epilepsy than the controls. Previous research has provided evidence to suggest that these factors are associated with poorer cognitive performance. ${ }^{4,16}$ Thus, any superiority shown by an untreated control group may be due to combination of three factors: low seizure frequency, short duration of epilepsy and no anticonvulsant medication.

Multivariate analysis demonstrated, however, that the test battery as a whole still discriminated between the groups when fit frequency was taken out as a covariate. Thus it may be concluded that the variance

Table 4 Significant correlation coefficients between tests scores and carbamazepine and carbamazepine 10,11 epoxide ( $C B Z-E)$ concentrations

\begin{tabular}{|c|c|c|c|}
\hline Test & $\begin{array}{l}\text { Carbamazepine } \\
(\mathrm{mg} / \mathrm{l})\end{array}$ & $\begin{array}{l}C B Z-E \\
(m g / l)\end{array}$ & $\frac{C B Z-E}{\text { Carbamazepine }} \%$ \\
\hline $\begin{array}{l}\text { Performance IQ } \\
\text { Decision Time* } \\
\text { Choice Reaction }\end{array}$ & $-0.28 t$ & $\begin{array}{r}-0.29+ \\
0.37_{+}^{+}\end{array}$ & $0.33+$ \\
\hline $\begin{array}{l}\text { Time* } \\
\text { Finger Tapping } \\
\text { Threshold }\end{array}$ & $0.30 \dagger$ & $\begin{array}{r}0.43 \S \\
-0.28 \dagger\end{array}$ & $0 \cdot 35_{+}^{+}$ \\
\hline $\begin{array}{l}\text { Detection* } \\
\text { Digits Back } \\
\text { Visual Span } \\
\text { Paired } \\
\text { Association } \\
\text { Learning* }\end{array}$ & $0.67 \S$ & $\begin{array}{r}0.61 \S \\
-0.31 \dagger \\
-0.35_{t}^{+}\end{array}$ & \\
\hline
\end{tabular}



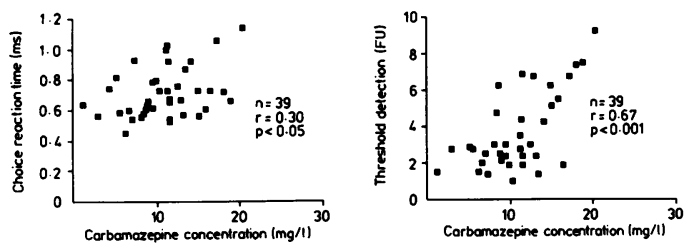

Fig 1 Correlations between choice reaction time and threshold detection with circulating carbamazepine concentration in 30 epileptic patients receiving the drug as monotherapy.

between the groups is not attributable to the effect of seizures per se.

(1) Comparison between monotherapy patients and untreated controls

The point of interest here is that the control group showed superiority on only one test: movement detection. The similarity between epileptic controls and carbamazepine monotherapy patients despite their higher seizure frequency allows the inference to be drawn that carbamazepine produced negligible cognitive impairment. This is in accordance with previous findings by other workers. ${ }^{9,11,17}$ Nevertheless, patients who develop severe psychomotor impairment with carbamazepine are unlikely to tolerate the drug long-term and so will exclude themselves from study.

(2) Comparison between monotherapy and polypharmacy patients

Carbamazepine monotherapy and polypharmacy patients were well matched for seizure frequency and duration of epilepsy. The monotherapy group was found to be superior in performance IQ, forward and backward digit span and both sedation and side effect scores. Patients were not randomly allocated to the treatment conditions and so it cannot be concluded that the cause of these differences lies purely in their treatment; some unmeasured factor cannot be excluded. However, the results do confirm that in a clinical population where therapies have not been determined by experimental design, there are real differences between patients taking carbamazepine alone and those taking it in combination with another drug. This finding supports a deleterious effect of polypharmacy on overall cerebral function. ${ }^{8,9,18}$

The active metabolite, CBZ-E, has anticonvulsant efficacy similar to the parent compound in animal epilepsy models ${ }^{19}$ and is more potent than carbamazepine in the treatment of trigeminal neuralgia. ${ }^{20}$ CBZ-E is also known to contribute to carbamazepine neurotoxicity. ${ }^{21}$ In this study, CBZ-E levels in the polypharmacy group were almost double those of the monotherapy patients with equivalent carbamazepine concentrations. This association is well known ${ }^{22-23}$ and is related to induction of carbamazepine epoxidation by phenytoin and phenobarbitone ${ }^{22}$ and inhibition of CBZ-E breakdown by sodium valproate. ${ }^{24}$ Thus, CBZ-E may have contributed substantially to the psychomotor impairment experienced by the polypharmacy patients in this study.

\section{(3) Concentration-effect relationships}

Performance on four and seven tests deteriorated with increasing carbamazepine and CBZ-E concentrations respectively. Andrews et al ${ }^{11}$ found a relationship between circulating carbamazepine levels and performance on a tracking task. In view of the inter-individual variation in response to these tests, large groups of patients require to be studied over a wide range of carbamazepine concentrations which may explain the inability of others to document such a clear-cut concentration-effect relationship. ${ }^{14,25}$

It is worth noting that more tests showed a
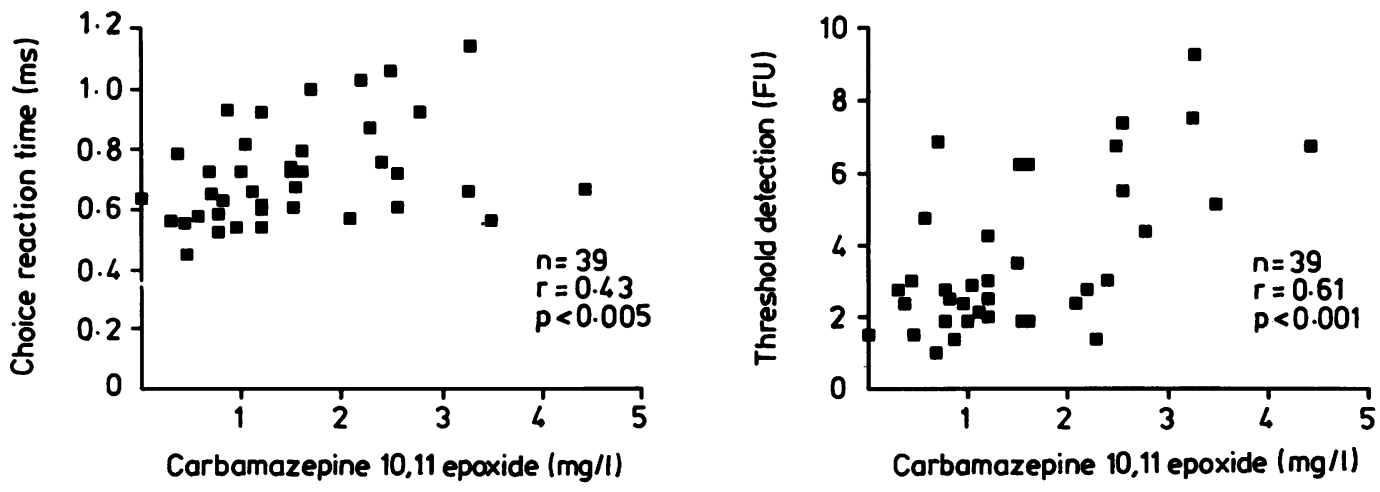

Fig 2 Correlations between choice reaction time and threshold detection with circulating carbamazepine 10,11 epoxide concentration in 40 epileptic patients receiving carbamazepine monotherapy. 
significant correlation with CBZ-E than with total carbamazepine. This suggests that CBZ-E may be more important in producing performance decrement. If cognitive impairment were solely attributable to the effects of CBZ-E on cerebral function, the ratio of CBZ-E to total carbamazepine would be expected to be a more sensitive predictor of dysfunction than total carbamazepine. That it was not supports an inciependent effect of both carbamazepine and its metabolite in producing psychomotor impairment. The term "impairment" in this context is relative; the carbamazepine group performed significantly worse than an appropriate control group on only one task. Nevertheless, the general trend was towards poorer performance with increasing carbamazepine and CBZ-E concentrations.

Care must be taken in inferring cause and effect between significant correlations; the relationship between any given pair of variables may be indirect, with a third factor "causing" both. Severity of epilepsy may lead to increased prescribing of carbamazepine and to increased cognitive impairment. Fit frequency, a reasonable measure of severity, did not correlate with total carbamazepine concentration. It did however appear to relate to CBZ-E level and it is possible that patients with higher CBZ-E levels had a more severe seizure disorder.

The division of the sample into high and low concentration groups served to identify the most discriminative tasks; for carbamazepine: threshold detection, finger tapping, decision and choice reaction times; for CBZ-E: threshold detection, decision and choice reaction times. It is clear that these tasks require relatively little information processing and have a strong visuo-motor component. It appears that the more primitive aspects of neuropsychological function are most affected by carbamazepine and its metabolite. These tasks may be of value in clinical practice to identify early those patients who are likely to become intolerant to high carbamazepine dosage.

We are grateful to Mrs Kay Cockburn for expert secretarial assistance and the Epilepsy Association of Scotland for financial support.

\section{References}

1 Lennox WG, Lennox MA. Epilepsy and Related Disorders. 1960; Boston: Little, Brown.

2 Pond DA, Bidwell BH. A survey of epilepsy in fourteen general practices. II Social and psychological aspects. 1960. Epilepsia 1:285-99.

3 Smith DB, Craft BR, Collins J, Mattson RH, Cramer JA. The VA Cooperative Study Group 118. Behavioural characteristics of epilepsy patients compared with normal controls. Epilepsia 1986;27:760-8.

4 Dodrill CE. Correlates of generalised tonic-clonic seizures with intellectual, neuropsychological, emotional and social function in patients with epilepsy. Epilepsia 1968;27:399-411.

5 Beran RG, Flanagan PJ. Psychological sequelae of epilepsy. The role of associated cerebral pathology. Epilepsia 1987;28: 107-10.

6 Reynolds EH, Travers RD. Serum anticonvulsant concentrations in epileptic patients with mental symptoms. Br J Psychiatry 1974;124:440-5.

7 Thompson PJ, Trimble MR. Anticonvulsant drugs and cognitive functions. Epilepsia 1982;23:531-44.

8 Ludgate J, Keating J, O'Dwyer R, Callaghan N. An improvement in cognitive function following polypharmacy reduction in a group of epileptic patients. Acta Neurol Scand 1985; 71:448-52.

9 Trimble MR, Thompson PJ, Huppert F. Anticonvulsant drugs and cognitive abilities. In: Canger R, Angeleri F, Penry JK. eds. Advances in Epileptology. New York, Raven Press, 1980; 199-204.

10 Novelly RA, Schwartz MM, Mattson RH, Cramer JA. Behavioural toxicity associated with antiepileptic drugs: Concepts and methods of assessment. Epilepsia 1986;27:331-40.

11 Andrewes DG, Bullen L, Tomlinson L, Elwes RDC, Reynolds EH. A comparative study of the cognitive effects of phenytoin and carbamazepine in new referrals with epilepsy. Epilepsia 1986;27:128-34.

12 Macphee GJA, Goldie C, Roulston D, Potter L, Agnew E, Brodie MJ. Effect of carbamazepine on psychomotor function in naive subjects. Eur J Clin Parmacol 1986;30:37-42.

13 Macphee GJA, McPhail EM, Butler E, Brodie MJ. Controlled evaluation of a supplementary dose of carbamazepine on psychomotor function in epileptic patients. Eur $J$ Clin Pharmacol 1986;31:195-9.

14 Brodie MJ, McPhail E, Macphee GJA, Larkin JG, Gray JMB. Psychomotor impairment and anticonvulsant therapy in adult epileptic patients. Eur J Clin Pharmacol 1987;31:655-60.

15 Meijer JWA. Anticonvulsant drugs: analytical techniques. In Richens A, Marks V, eds. Therapeutic Drug Monitoring. Edinburgh, Churchill Livingstone, 1981;349-69.

16 Hodgman CH, McAnarney ER, Myers GJ et al. Emotional complications of adolescent grand mal epilepsy. $J$ Pediatr 1979;95:309-12.

17 Dodrill CB, Troupin AS. Psychotropic effects of carbamazepine in epilepsy: a double blind comparison with phenytoin. Neurology 1977;27:1023-8.

18 Fischbacher E. Effect of reduction of anticonvulsants on wellbeing. Br Med J 1982;285:423-4.

19 Morselli PL, Gerna M, de Maio D, Zanda G, Viani F, Gerattini S. Pharmacokinetic studies on carbamazepine in volunteers and in epileptic patients. In: Schreider H, Janz D, Gardner-Thorpe C, Meinardi H, Sherwin AL. Pharmacology of Anti-epileptic Drugs. Berlin, Springer-Verlag, 1975;166-80.

20 Tomson T, Bertilsson L. Potent therapeutic effect of carbamazepine-10, 11-epoxide in trigeminal neuralgia. Arch Neurol 1984; 41:598-602.

21 Patsalos PN, Stephenson TJ, Krishna S, Elyas AA, Lascelles PT, Wiles CM. Side-effects induced by carbamazepine 10,11 epoxide. Lancet 1985;ii:496.

22 McKauge L, Tyrer JH, Eadie MJ. Factors influencing simultaneous concentrations of carbamazepine and its epoxide in plasma. Ther Drug Monit 1981;3:69-70.

23 Brodie MJ, Forrest G, Rapeport WG. Carbamazepine 10,11 epoxide concentrations in epileptics on carbamazepine alone and in combination with other anticonvulsants. $\mathrm{Br} \mathrm{J}$ Clin Pharmacol 1983;16:747-50.

24 Macphee GJA, Mitchell J, Wiseman L, et al. Effect of sodium valproate on carbamazepine disposition and psychomotor profile in man. Br J Clin Pharmacol 1988;25:59-66.

25 Post RM, Uhde TW, Ballenger JC, Chatterji DC, Greene CF, Bunney WE. Carbamazepine and its 10,11 epoxide metabolite in plasma and CSF. Arch Gen Psychiat 1983;40:673-6. 